1996-09-01

\title{
Bounding Elastic Constants of an Orthotropic Polycrystal using Measurements of the Microstructure
}

Brent L. Adams

b_I_adams@byu.edu

M. J. Beran

T. A. Mason

Ted Olsen

Follow this and additional works at: https://scholarsarchive.byu.edu/facpub

Part of the Mechanical Engineering Commons

Original Publication Citation

J. Mech. Phys. Solids, Vol. 44, No. 9, pp. 1543-1563, 1996.

\section{BYU ScholarsArchive Citation}

Adams, Brent L.; Beran, M. J.; Mason, T. A.; and Olsen, Ted, "Bounding Elastic Constants of an Orthotropic Polycrystal using Measurements of the Microstructure" (1996). Faculty Publications. 1145.

https://scholarsarchive.byu.edu/facpub/1145

This Peer-Reviewed Article is brought to you for free and open access by BYU ScholarsArchive. It has been accepted for inclusion in Faculty Publications by an authorized administrator of BYU ScholarsArchive. For more information, please contact ellen_amatangelo@byu.edu. 
http://www.sciencedirect.com/science/journal/00225096

\title{
BOUNDING ELASTIC CONSTANTS OF AN ORTHOTROPIC POLYCRYSTAL USING MEASUREMENTS OF THE MICROSTRUCTURE
}

\author{
M. J. BERAN \\ Faculty of Engineering, Tel Aviv University, Ramat Aviv, Israel \\ T. A. MASON \\ Lockheed Idaho Technologies Co., P.O. Box 1625, Idaho Falls, ID 83415, U.S.A. \\ B. L. ADAMS \\ Department of Materials Science and Engineering, Carnegie Mellon University, Pittsburgh, PA 15213, \\ U.S.A. \\ and \\ T. OLSEN \\ Department of Mathematics, Michigan Technological University, Houghton, MI, U.S.A.
}

\begin{abstract}
We show how information about the elastic stiffness and compliance of an orthotropic polycrystal may be obtained from measurements of the statistical properties of the microstructure. We begin by discussing the statistical properties of the governing equations and the hierarchy that results when the equations are averaged. Perturbation solutions are obtained in terms of low-order statistical information. Using methods previously developed, we derive bounds for the elastic stiffnesses and compliances. A hierarchy of bounds is derived using the statistical information obtained from measurement of the microstructure. We discuss the methods used to obtain the correlation functions of the microstructure and provide a specific example, using data obtained from a copper sample. Copyright $C 1996$ Elsevier Science Ltd
\end{abstract}

Keywords: A. microstructures, B. constituitive behavior, C. polycrystalline material, D. electron microscopy.

\section{INTRODUCTION}

The basic equations governing the static linear elastic properties of a medium with elastic constants that vary with position are 


$$
\partial \tau_{i j} / \partial x_{j}=0,
$$

where

$$
\tau_{i j}(\mathbf{x})=C_{i j k m}(\mathbf{x}) e_{k m}(\mathbf{x})
$$

and

$$
e_{i j}=(1 / 2)\left[\partial u_{i} / \partial x_{j}+\partial u_{j} / \partial x_{i}\right] .
$$

Here $\tau_{i j}$ is the stress tensor, $e_{i j}$ is the strain tensor and $u_{i}$ is the displacement field. The elastic stiffnesses are represented by the fourth-order tensor $C_{i j k m}$. The fourthorder compliance tensor is derived from the relation

$$
C_{i j k m} S_{k m p q}=(1 / 2)\left[\delta_{i p} \delta_{j q}+\delta_{i q} \delta_{j p}\right] .
$$

Using the compliance tensor we may write

$$
e_{i j}=S_{i j k m} \tau_{k m} .
$$

When $C_{i j k m}$ is a statistically homogeneous random function, as is often the case in a polycrystal, it is natural to ask if the macroscopic elastic properties of the polycrystal may be described by equations similar to those of $(1 \mathrm{a}-\mathrm{c})$, but with the stress and strain fields replaced by average quantities $\left\langle\tau_{i j}\right\rangle$ and $\left\langle e_{i j}\right\rangle$ and the elastic tensor by an effective tensor $C_{i j k \mathrm{~m}}^{*}$. The brackets represent an ensemble average over many realizations of the random microstructure. That is, we wish to replace $(1 \mathrm{a}-\mathrm{c})$ by the set

$$
\begin{gathered}
\partial\left\langle\tau_{i j}\right\rangle / \partial x_{j}=0, \\
\left\langle\tau_{i j}\right\rangle=C_{i j k m}^{*}\left\langle e_{k m}\right\rangle
\end{gathered}
$$

and

$$
\left\langle e_{i j}\right\rangle=(1 / 2)\left[\partial\left\langle u_{i}\right\rangle / \partial x_{j}+\partial\left\langle u_{j}\right\rangle / \partial x_{i}\right] .
$$

The above set of equations may be used when all the characteristic correlation lengths associated with the statistical properties of the microstructure are small compared to lengths associated with the sample size and to any characteristic lengths associated with the variation in forcing functions. We refer the readers to Brown (1965), Beran (1968), Kroner (1967, 1986), Korringa (1973), McCoy (1981) and Willis (1981) for basic references on the subject. The difficulty in using ( $4 a-c)$ is in determining $C_{i j k m}^{*}$.

The constants in the tensor $C_{i j k m}^{*}$ may be determined experimentally just as the constants of $C_{i j k m}$ for a single crystal were determined by Ledbetter and Read (1977). However, the tensor $C_{i j k m}^{*}$ is completely determined from a knowledge of the single crystal tensor $C_{i j k m}$ and the statistical properties of the microstructure (i.e. the shape and arrangement statistics of the individual crystals). The advantage of determining $C_{i j k m}^{*}$ from the statistical properties of the microstructure is that if these properties can be correlated with the manufacturing process then the possibility arises for creating materials with predetermined elastic properties.

Our purpose in this paper is to discuss the relationship between the effective constants $C_{i j k m}^{*}$ and $S_{i j k m}^{*}$ and the statistical properties of the random fields $C_{i j k m}(\mathbf{x})$ and $S_{i j k m}(\mathbf{x})$ in orthotropic polycrystals. In the next section we will discuss the existing 
formal relations and show that, in principle, it is necessary to know all the correlation functions associated with $C_{i j k m}(\mathbf{x})$ in order to determine $C_{i j k m}^{*}$ and $S_{i j k m}^{*}$. In Section 3, perturbation solutions will be given. In Section 4, we will show that bounds on the effective constants may be obtained from incomplete statistical information and various level bounds will be presented. The relation of the theoretical work of this paper to a parallel experimental program on the statistical properties of the microstructure will be discussed in Section 5 and theoretical-experimental comparisons will be given.

\section{DETERMINATION OF $C_{i, k m}^{*}$ AND $S_{i j k m}^{*}$ FROM THE STATISTICAL PROPERTIES OF $C_{i j k m}$}

In this section, we shall derive formulas for $C_{i j k m}^{*}$ and $S_{i, k m}^{*}$ in terms of the correlation functions of the polycrystal's microstructure. The functions $C_{i j k m}(\mathbf{x})$ and $S_{i j k m}(\mathbf{x})$ are conveniently written in terms of their mean values and a spatially fluctuating part. We write

$$
\begin{aligned}
& C_{i j k m}(\mathbf{x})=\left\langle C_{i j k m}\right\rangle+C_{i j k m}^{\prime}(\mathbf{x}), \\
& S_{i j k m}(\mathbf{x})=\left\langle S_{i j k m}\right\rangle+S_{i j k m}^{\prime}(\mathbf{x}),
\end{aligned}
$$

where

$$
\begin{aligned}
& \left\langle C_{i j k m}^{\prime}(\mathbf{x})\right\rangle=0, \\
& \left\langle S_{i j k m}^{\prime}(\mathbf{x})\right\rangle=0 .
\end{aligned}
$$

The stress, strain and displacement fields are similarly written as

$$
\begin{aligned}
& \tau_{i j}(\mathbf{x})=\left\langle\tau_{i j}\right\rangle+\tau_{i j}^{\prime}(\mathbf{x}), \\
& e_{i j}(\mathbf{x})=\left\langle e_{i j}\right\rangle+e_{i j}^{\prime}(\mathbf{x}), \\
& u_{i}(\mathbf{x})=\left\langle u_{i}(\mathbf{x})\right\rangle+u_{i}^{\prime}(\mathbf{x}) .
\end{aligned}
$$

Here, $\left\langle\tau_{i j}\right\rangle$ and $\left\langle e_{i j}\right\rangle$ are independent of position and $\left\langle u_{i}(\mathbf{x})\right\rangle$ is a linear function of position. The average values of the fluctuating parts are equal to zero.

From the relation

$$
\left\langle\tau_{i j}\right\rangle=\left\langle C_{i j k m}(\mathbf{x}) e_{k m}(\mathbf{x})\right\rangle
$$

we find, using (5), (6) and (7)

$$
\left\langle\tau_{i j}\right\rangle=\left\langle C_{i j k m}\right\rangle\left\langle e_{k m}\right\rangle+\left\langle C_{i j k m}^{\prime}(\mathbf{x}) e_{k m}^{\prime}(\mathbf{x})\right\rangle .
$$

From (4b), we then find

$$
C_{i j k m}^{*}\left\langle e_{k m}\right\rangle=\left\langle C_{i j k m}\right\rangle\left\langle e_{k m}\right\rangle+\left\langle C_{i j k m}^{\prime}(\mathbf{x}) e_{k m}^{\prime}(\mathbf{x})\right\rangle .
$$

From $(10)$, it is seen that, in order to determine the effective constants $C_{i j k m}^{*}$, we must have a knowledge of the correlation functions $\left\langle C_{i j k m}^{\prime}(\mathbf{x}) e_{k m}^{\prime}(\mathbf{x})\right\rangle$. The correlation can only be determined by solving the governing equations given in (1). As we now show, this requires a knowledge of all the statistical moments associated with the 
fluctuation field $C_{i j k m}^{\prime \prime}(\mathbf{x})$. Substituting (5) into (1) we find, after some manipulation, (note that $C_{i j k m}^{\prime}=C_{i j m k}^{\prime}$ )

$$
\left\langle C_{i j k m}\right\rangle \partial^{2} u_{k}^{\prime}(\mathbf{x}) / \partial x_{j} \partial x_{m}+\partial / \partial x_{j}\left[C_{i j k m}^{\prime}(\mathbf{x}) e_{k m}(\mathbf{x})\right]=0 .
$$

Equation (11) can be written as an integral equation using the Green's function defined by the equation

$$
\left\langle C_{i j k m}\right\rangle \partial^{2} G_{k p}\left(\mathbf{x}, \mathbf{x}^{\prime}\right) / \partial x_{j} \partial x_{m}+\delta_{i p} \delta\left(\mathbf{x}-\mathbf{x}^{\prime}\right)=0
$$

(see Willis, 1981 and Barnett, 1972):

$$
u_{k}^{\prime}(\mathbf{x})=\int_{V} G_{k p}\left(\mathbf{x}, \mathbf{x}^{\prime}\right) \partial\left[C_{p m r s}^{\prime}\left(\mathbf{x}^{\prime}\right) e_{r s}\left(\mathbf{x}^{\prime}\right)\right] / \partial x_{m}^{\prime} \mathrm{d} \mathbf{x}^{\prime} .
$$

We next differentiate (13) to obtain an expression for $e_{k u}^{\prime}(\mathbf{x})$. Multiplying the resulting equation by $C_{i j k u}(\mathbf{x})$, and averaging, we obtain finally

$$
\begin{aligned}
\left\langle C_{i j k u}^{\prime}(\mathbf{x}) e_{k u}^{\prime}(\mathbf{x})\right\rangle= & \int_{V} K_{k p u}\left(\mathbf{x}, \mathbf{x}^{\prime}\right)\left[\partial\left\langle C_{i j k u}^{\prime}(\mathbf{x}) C_{p m r s}^{\prime}\left(\mathbf{x}^{\prime}\right)\right\rangle / \partial x_{m}^{\prime}\right]\left\langle e_{r s}\right\rangle \mathrm{d} \mathbf{x}^{\prime} \\
& +\int_{V} K_{k p u}\left(\mathbf{x}, \mathbf{x}^{\prime}\right)\left[\partial\left\langle C_{i j k u}^{\prime}(\mathbf{x}) C_{p m r s}^{\prime}\left(\mathbf{x}^{\prime}\right) e_{r s}\left(\mathbf{x}^{\prime}\right)\right\rangle / \partial x_{m}^{\prime}\right] \mathrm{d} \mathbf{x}^{\prime}
\end{aligned}
$$

where

$$
K_{k p u}=(1 / 2)\left[G_{k p, k}+G_{u p, k}\right] .
$$

(The comma in the subscript of $G$ denotes differentiation.)

Unfortunately (14) cannot be used to determine $\left\langle C_{i j k u}^{\prime}(\mathbf{x}) e_{k u}^{\prime}(\mathbf{x})\right\rangle$ from the statistical properties of $C_{i j k u}^{\prime}(\mathbf{x})$ since it contains the unknown expression

$$
\left\langle C_{i j k u}^{\prime}(\mathbf{x}) C_{p m r s}^{\prime}\left(\mathbf{x}^{\prime}\right) e_{r s}\left(\mathbf{x}^{\prime}\right)\right\rangle \text {. }
$$

An expression for this three-point correlation function could be obtained from (13), but it in turn would contain an unknown four-point correlation function. Formally, an entire hierarchy, containing successively higher moments, could be constructed which would represent the solution to the statistical problem. Beran (1968) showed that from a mathematical point of view, this infinite hierarchy would be equivalent to a functional equation governing the $n$-point joint probability density function between the strain and elastic constant fields in the limit as $n$ approaches infinity.

From a practical point of view, the statistical hierarchy is only of use if it can be terminated in some reasonable manner. Standard perturbation theory is perhaps the simplest cutoff procedure and this theory is discussed in Section 3. Other cutoff procedures have been used, but none have validity for a wide variety of materials. Instead, use has been made of variational principles which bound the values of the elastic constants rather than determining them exactly. At present, in light of the difficulty of obtaining useful approximations for terminating the hierarchy, bounding methods are extensively used. We shall discuss these procedures in Section 4. 


\section{PERTURBATION THEORY}

The use of perturbation theory is based on the assumption that the magnitude of the fluctuations in the elastic constants $C_{i j k m}^{\prime}(\mathbf{x})$ is much smaller than the magnitudes of the mean values $\left\langle C_{i j k m}\right\rangle$. It is difficult to determine exactly what is meant by small, but, in Section 5, we shall present experimental results that show how the perturbation result compares to experimental results in a typical case.

When the fluctuations are small, the second term on the right-hand side of (14) may be neglected and we obtain the approximation

$$
\left\langle C_{i j k u}^{\prime}(\mathbf{x}) e_{k u}^{\prime}(\mathbf{x})\right\rangle=\int_{V} K_{k p u}\left(\mathbf{x}, \mathbf{x}^{\prime}\right)\left[\partial\left\langle C_{i j k u}^{\prime}(\mathbf{x}) C_{p m r s}^{\prime}\left(\mathbf{x}^{\prime}\right)\right\rangle / \partial x_{m}^{\prime}\right]\left\langle e_{r s}\right\rangle \mathrm{d} \mathbf{x}^{\prime} .
$$

This expression allows us to determine the elastic constants in terms of the derivative of the two-point correlation function $\left\langle C_{i j k u}^{\prime}(\mathbf{x}) C_{p m r s}^{\prime}\left(\mathbf{x}^{\prime}\right)\right\rangle$. To obtain a more accurate solution, (14) may be iterated and an additional term, containing the three-point correlation function, added to the right-hand side of (15).

The derivative of the correlation function is difficult to determine experimentally and it is convenient to integrate the integral by parts. Integrating over a spherical annulus, with the inner radius approaching zero and the outer radius approaching infinity, we obtain

$$
\left\langle C_{i j k u}^{\prime}(\mathbf{x}) e_{k u}^{\prime}(\mathbf{x})\right\rangle=M_{i j}-\int_{V} K_{k p u m}\left(\mathbf{x}, \mathbf{x}^{\prime}\right)\left\langle C_{i j k u}^{\prime}(\mathbf{x}) C_{p m r s}^{\prime}\left(\mathbf{x}^{\prime}\right)\right\rangle\left\langle e_{r_{s}}\right\rangle \mathrm{d} \mathbf{x}^{\prime},
$$

where

$$
K_{k p u m}=(1 / 2)\left[G_{k p, u m}+G_{u p, k m}\right]
$$

and

$$
M_{i j}=\int_{V} \partial\left[K_{k p u}\left(\mathbf{x}, \mathbf{x}^{\prime}\right)\left\langle C_{i j k u}^{\prime}(\mathbf{x}) C_{p m r s}^{\prime}\left(\mathbf{x}^{\prime}\right)\right\rangle\right] / \partial x_{m}^{\prime}\left\langle e_{r s}\right\rangle \mathrm{d} \mathbf{x}^{\prime} .
$$

The expression for $M_{i j}$ can be converted to a surface integral by applying Gauss' theorem. The resulting surface integral requires evaluation on a surface at infinity and on a surface enclosing the singularity in $K_{k p u}$. Choosing both surfaces as spheres, it can easily be shown that the sphere at infinity yields no contribution since it is assumed that the correlation between $C_{i j k u}^{\prime}(\mathbf{x})$ and $C_{p m r s}^{\prime}\left(\mathbf{x}^{\prime}\right)$ goes rapidly to zero as the distance between $\mathbf{x}$ and $\mathbf{x}^{\prime}$ approaches infinity. The sphere surrounding the finite singularity gives a contribution which depends on the first derivative of the Green's function and the function $\left\langle C_{i j k u}^{\prime}(\mathbf{x}) C_{p m r s}^{\prime}(\mathbf{x})\right\rangle$.

The results of evaluating (16) for an orthotropic polycrystal (cold-rolled copper) will be given in Section 5 and compared to experimental results.

\section{BOUNDS ON THE ELASTIC STIFFNESS AND COMPLIANCE}

The bounds we discuss in this section are found from two classical variational principles. We note that the average energy can be written in the following forms 


$$
\begin{aligned}
U & =(1 / 2)\left\langle e_{i j} \tau_{i j}\right\rangle \\
& =(1 / 2) C_{i j k m}^{*}\left\langle e_{i j}\right\rangle\left\langle e_{k m}\right\rangle \\
& =(1 / 2) S_{i j k m}^{*}\left\langle\tau_{i j}\right\rangle\left\langle\tau_{k m}\right\rangle,
\end{aligned}
$$

where we invoke the ergodic hypothesis to equate the ensemble average $\langle>$ with the volume average. That is

$$
2 U=\left\langle e_{i j}(\mathbf{x}) \tau_{i j}(\mathbf{x})\right\rangle=\lim (V \rightarrow \infty)(1 / V) \int_{V} e_{i j}(\mathbf{x}) \tau_{i j}(\mathbf{x}) \mathrm{d} \mathbf{x} .
$$

Equation (la) may be derived from the principle of minimum potential energy. This is stated as follows. The expression for $U$ in (19), subject to the subsidiary conditions in (1c), is stationary and assumes a minimum value for strains satisfying (la) and (lb). For a material sample confined by a boundary surface, we require that the variation of $u_{i}$ be zero on the boundary. For an infinite ensemble of samples we require that the mean value of any trial function $e_{A i j}(\mathbf{x})$, be equal to the mean value of the true solution. We then find that

$$
C_{i j k m}^{*}\left\langle e_{i j}\right\rangle\left\langle e_{k m}\right\rangle \leqslant\left\langle C_{i j k m}(\mathbf{x}) e_{A i j}(\mathbf{x}) e_{A k m}(\mathbf{x})\right\rangle .
$$

Equation (1) may also be derived from the principle of complementary energy. Here the energy $U$, defined in (19), subject to the subsidiary conditions of (1a) and $\tau_{i j}=\tau_{i j}$, is stationary and a minimum for stresses satisfying (3) and (1c). We require, here, that the variations of the tractions are zero on the bounding surface or that the mean value of the trial solution $\tau_{A i j}(\mathbf{x})$ is equal to the true mean value. We then find

$$
S_{i j k m}^{*}\left\langle\tau_{i j}\right\rangle\left\langle\tau_{k m}\right\rangle \leqslant\left\langle S_{i j k m}(\mathbf{x}) \tau_{A i j}(\mathbf{x}) \tau_{A k m}(\mathbf{x})\right\rangle .
$$

\subsection{Trial functions}

4.1.1. Constant trial functions. Perhaps the simplest trial functions that may be used are $e_{A i j}=\left\langle e_{i j}\right\rangle$ in (20) and $\tau_{A i j}=\left\langle\tau_{i j}\right\rangle$ in (21). We find

$$
\left\langle e_{i j}\right\rangle C_{i j k m}^{*}\left\langle e_{k m}\right\rangle \leqslant\left\langle e_{i j}\right\rangle\left\langle C_{i j k m}\right\rangle\left\langle e_{k m}\right\rangle
$$

and

$$
\left\langle\tau_{i j}\right\rangle S_{i j k m}^{*}\left\langle\tau_{k m}\right\rangle \leqslant\left\langle\tau_{i j}\right\rangle\left\langle S_{i j k m}\right\rangle\left\langle\tau_{k m}\right\rangle
$$

for any average applied strain $\left\langle e_{i j}\right\rangle$ or applied stress $\left\langle\tau_{i j}\right\rangle$.

It may be shown that (23) implies

$$
\left\langle e_{i j}\right\rangle\left(S_{i j k m}^{*}\right)^{-1}\left\langle e_{k m}\right\rangle \geqslant\left\langle e_{i j}\right\rangle\left(\left\langle S_{i j k m}\right\rangle\right)^{-1}\left\langle e_{k m}\right\rangle
$$

for any average strain $\left\langle e_{i i}\right\rangle$.

Using (22), (23) and (24) then yields

$$
\left\langle e_{i j}\right\rangle\left(\langle S\rangle^{-1}\right)_{i j k m}\left\langle e_{k m}\right\rangle \leqslant\left\langle e_{i j}\right\rangle C_{i, k m}^{*}\left\langle e_{k m}\right\rangle \leqslant\left\langle e_{i j}\right\rangle\left\langle C_{i j k m}\right\rangle\left\langle e_{k m}\right\rangle .
$$

There is a similar expression for $S_{i j k m}^{*}$.

Equation (25) does not provide bounds for all components of $C_{i j k m}^{*}$. It does, 
however, provide bounds for $C_{i i i i}$ (no sum on $i$ ) and $C_{i j i j}$ (no sum on $i$ or $j, i \neq j$ ). For an orthotropic crystal composed of cubic grains this is sufficient information since we note that in this case the bulk modulus

$$
k^{*}=(1 / 9) C_{i i k k}^{*}=1 / S_{i i k k}^{*}=(1 / 9) C_{i k k k}(\mathbf{x})
$$

(sum on $i$ and $k$ ) is independent of the sample's macroscopic symmetry and internal microstructure. Walpole (1987) has shown that only seven constants are required to completely specify the elastic tensor for an orthotropic polycrystal with cubic grains and Hashin and Shtrikman (1962) noted that the upper and lower bounds for the bulk modulus coincided for materials with cubic symmetry.

We note that for the shear components $C_{i j i j}^{*}$ (no sum on $i$ or $j$ and $i \neq j$ ) we have the relation

$$
C_{i j i j}^{*}=1 /\left(4 S_{i j i j}^{*}\right)
$$

The constants $S_{i i t i}^{*}$ (no sum on $i$ ) can be bounded in a similar manner to $C_{i i i i}^{*}$ (no sum on $i$ ) but the constants are not simply related.

Bounds for the constants using the constant trial functions are given in Section 5 and compared to experimental results.

4.1.2. Trial functions that depend on position. The bounds just obtained in Section 4.1.1 depend only upon the average of the functions $C_{i j k m}(\mathbf{x})$ and $S_{i j k m}(\mathbf{x})$. They do not depend upon higher-order statistical information. To obtain more restrictive bounds that depend upon the two- and three-point correlation functions of the elastic and compliance constants, we must introduce trial functions that depend upon position. A simple way to do this is to use the perturbation solutions obtained in Section 3 as trial functions (see Beran 1968).

We begin by obtaining bounds for the shear modulii $C_{i j i j}^{*}$ (no sum on $i$ and $j$ and $i \neq j$ ). As an example we treat $C_{1212}^{*}$ and consider a mean field where the only nonzero component is $\left\langle e_{12}\right\rangle$. As a trial function we use

$$
e_{A i j}(\mathbf{x})=\left\langle e_{i j}\right\rangle+\alpha \int_{V} K_{p i j}\left(\mathbf{x}, \mathbf{x}^{\prime}\right) \partial C_{p m 12}^{\prime}\left(\mathbf{x}^{\prime}\right) / \partial x_{m}^{\prime}\left\langle e_{12}\right\rangle \mathrm{d} \mathbf{x}^{\prime} .
$$

This expression is the first term of the perturbation solution multiplied by a constant. This trial function satisfies the condition $\left\langle e_{A 12}\right\rangle=\left\langle e_{i 2}\right\rangle$ and (1c). Upon substitution into (20) we find the inequality

$$
\begin{aligned}
C_{1212}^{*} \leqslant & \left\langle C_{1212}\right\rangle+2 \alpha\left\langle C_{12 k m}^{\prime}(\mathbf{x}) \hat{e}_{A k m}^{\prime}(\mathbf{x})\right\rangle+ \\
& \alpha^{2}\left[\left\langle C_{i j k m}\right\rangle\left\langle\hat{e}_{A i j}^{\prime}(\mathbf{x}) \hat{e}_{A k m}^{\prime}(\mathbf{x})\right\rangle+\left\langle C_{i j k m}^{\prime}(\mathbf{x}) \hat{e}_{A i j}^{\prime}(\mathbf{x}) \hat{e}_{A k m}^{\prime}(\mathbf{x})\right\rangle\right] /\left\langle e_{12}\right\rangle,
\end{aligned}
$$

where $\hat{e}_{A i j}^{\prime}(\mathbf{x})$ represents the integral term in (28). The term multiplying $\alpha$ and the first term multiplying $\alpha^{2}$ contain two-point correlation information while the second term multiplying $\alpha^{2}$ contains three-point correlation information.

The constant $\alpha$ is found by minimizing the right-hand side of (29) with respect to $x$. We find 


$$
C_{1212}^{*} \leqslant\left\langle C_{1212}\right\rangle-J_{S 1}^{2} / J_{S 2}
$$

where

$$
J_{S 1}=\left\langle C_{12 k m}^{\prime}(\mathbf{x}) \hat{e}_{A k m}^{\prime}(\mathbf{x})\right\rangle\left\langle\left\langle e_{12}\right\rangle\right.
$$

and

$$
J_{S 2}=\left\langle C_{i j k m}(\mathbf{x}) \hat{e}_{A i j}^{\prime}(\mathbf{x}) \hat{e}_{A k m}^{\prime}(\mathbf{x})\right\rangle /\left\langle e_{12}\right\rangle^{2} .
$$

A similar procedure may be used to find a lower bound for $C_{1212}^{*}$, since $S_{1212}^{*}$ and $C_{1212}^{*}$, are related by the expression given in (27). The upper bound for $S_{1212}^{*}$ is found using (21) and the trial function

$$
\tau_{A i j}(\mathbf{x})=\left\langle\tau_{i j}\right\rangle+\beta\left[\left\langle C_{i j k m}\right\rangle e_{A k m}^{\prime}(\mathbf{x})+C_{i j 12}^{\prime}(\mathbf{x})\left\langle e_{12}\right\rangle\right] .
$$

After some manipulation we find

$$
C_{1212}^{*} \geqslant(1 / 4)\left[1 /\left\langle S_{1212}\right\rangle-K_{S 1}^{2} / K_{S 2}\right],
$$

where

$$
K_{S 1}=\left\langle S_{12 k m}^{\prime}(\mathbf{x}) \tau_{A k m}^{\prime}(\mathbf{x})\right\rangle\left\langle\left\langle\tau_{12}\right\rangle\right.
$$

and

$$
K_{S 2}=\left\langle S_{i j k m}(\mathbf{x}) \tau_{A i j}^{\prime}(\mathbf{x}) \tau_{A k m}^{\prime}(\mathbf{x})\right\rangle\left\langle\left\langle\tau_{12}\right\rangle^{2}\right.
$$

Here

$$
\tau_{A i j}^{\prime}(\mathbf{x})=\tau_{A i j}(\mathbf{x})-\left\langle\tau_{i j}\right\rangle .
$$

The bounds for $C_{1313}^{*}$ and $C_{2323}^{*}$ are obtained in a similar manner.

The upper bound for the quantities like $C_{i i i}^{*}$ (no sum on $i$ ) are obtained using an expression similar to (28). For $C_{1111}^{*}$ the only non-zero component of the strain field is $\left\langle e_{11}\right\rangle$ and the "12" subscripts in (28) are replaced by " 11 ". The final expression is

$$
C_{111}^{*} \leqslant\left\langle C_{1111}\right\rangle-J_{C 1}^{2} / J_{C 2} \text {. }
$$

Here $J_{C 1}$ and $J_{C 2}$ are found from $J_{S 1}$ and $J_{S 2}$, respectively, by changing the "12" subscripts to " $11 "$ ".

An upper bound for $S_{1111}^{*}$ can be found by using the trial function given in (33) and replacing $\left\langle e_{i j}\right\rangle$ by $\left\langle S_{i j k m}\right\rangle\left\langle\tau_{k m}\right\rangle$. Unfortunately the lower bound for $C_{1111}^{*}$ is not simply obtainable from the upper bound of $S_{1111}^{*}$. Rather we require bounds on several components of the tensor $S_{i j k m}^{*}$ in order to invert the elastic tensor. In view of the complexity of the calculations, we do not consider them here.

4.1.3. Bounds that depend only on the two-point correlation function. The bounds given in the previous section depend on both the two- and three-point correlation functions. Kroner (1977) and Willis (1981) have shown that bounds may be derived that depend only upon the two-point correlation function. A direct way to do this is to use the variational principle used by Hashin and Shtrikman (1962). In this section, we shall show how the two-point upper bound corresponding to (30) is derived from (30). 
To derive the two-point upper bound for $C_{1212}^{*}$, we replace $C_{p m 12}^{\prime}$ in (28) by $\delta C_{p m 12}(\mathbf{x})=C_{p m 12}(\mathbf{x})-C_{p m 12}^{\mathbf{R}}$, where $C_{p m 12}^{\mathrm{R}}$ is a constant reference tensor. We also require that the tensor $\delta C_{i, k m}(\mathbf{x})$ is negative definite. The upper bound given in (30) remains the same except that $C_{i j k m}^{\prime}(\mathbf{x})$ is replaced by $\delta C_{i j k m}(\mathbf{x})$. In order to obtain an upper bound that is independent of the three-point correlation function that appears in the expression

$$
\left\langle\delta C_{i j k m}(\mathbf{x}) e_{A i j}^{\prime}(\mathbf{x}) e_{A k m}^{\prime}(\mathbf{x})\right\rangle
$$

we require an upper bound for this expression in terms of the two-point correlation function. This bound may be obtained using the Schwartz inequality. We find

$$
\left\langle\delta C_{i j k m}(\mathbf{x}) e_{A i j}^{\prime}(\mathbf{x}) e_{A k m}^{\prime}(\mathbf{x})\right\rangle \leqslant-\left\langle\delta C_{12 k m}(\mathbf{x}) e_{A k m}^{\prime}(\mathbf{x})\right\rangle^{2}\left\langle\left\langle\delta C_{1212}\right\rangle .\right.
$$

The final expression for the upper bound is then

$$
C_{1212}^{*} \leqslant C_{1212}^{\mathrm{R}}+\left\langle\delta C_{1212}\right\rangle^{2} /\left[\left\langle\delta C_{1212}\right\rangle+\left\langle\delta C_{12 k m}(\mathbf{x}) \hat{e}_{A k m}^{\prime}(\mathbf{x})\right\rangle\right] .
$$

The lower bound for $C_{1212}^{*}$ and the other bounds that depend upon one and twopoint correlation information are most easily derived using the Hashin-Shtrikman approach. The lower bound for $C_{1212}^{*}$ is found to be

$$
C_{1212}^{*} \geqslant C_{1212}^{R}+\left\langle\delta C_{1212}\right\rangle^{2} /\left[\left\langle\delta C_{1212}\right\rangle+\left\langle\delta C_{12 k m}(\mathbf{x}) \hat{e}_{A k m}^{\prime}(\mathbf{x})\right\rangle\right],
$$

where now $\delta C_{i j k m}(\mathbf{x})$ is positive definite. We note that in the limit of very small fluctuations the upper and lower bounds coincide with the perturbation solution given in (16).

4.1.4. Importance of the shape of the two-point correlation function. We pointed out in Section 3 (the perturbation solution) that the contribution to the effective constant depends upon a part $M_{i j}$ that is independent of the shape of the correlation function $\left\langle C_{i j k u}^{\prime}(\mathbf{x}) C_{p m r s}^{\prime}\left(\mathbf{x}^{\prime}\right)\right\rangle$ and a remaining volume integral that depends upon the shape. If the statistics of the tensor $C_{i j k m}^{\prime}$ are isotropic, it may be shown that the volume integral in (16) is identically zero (see Beran, 1968). In the case of non-isotropic statistics, the volume integral is non-zero but if the statistics are almost isotropic, we expect the $M_{i j}$ contribution to be dominant.

In Section 5, we consider annealed copper plate and we shall show that in this case the $M_{i j}$ contribution is indeed the dominant contribution in the perturbation solution. In addition, Mason (1994) has considered the bounds given in Section 4.1.3 and similar bounds derived using a generalization of the Hashin-Shtrikman approach. The bounds for this material are relatively insensitive to the shape of the twopoint correlation function. The bounds depend principally on the variance of the fluctuations (i.e. the value of the two-point correlation function when the separation of the two points is zero).

\section{COMPARISON OF EXPERIMENTAL DATA WITH THEORY}

\subsection{Background}

For crystalline materials the term microstructure refers to the myriad features describing the sizes, shapes and spatial placement of the crystalline components 
(grains and subgrains). Microstructure also refers to other aspects of the defect structure such as the state of dislocation. In this paper, both theoretical and practical considerations prescribe the main focus to be the low-order statistics of the spatial placement of the lattice orientation among grains and subgrains of the homophase polycrystal.

Linear elasticity of polycrystalline metals and ceramics is an example of "defectinsensitive properties" when grain and subgrain boundaries, whose properties differ markedly from those of the constituent crystallites, occupy negligible volume. In practical terms, since the typical range of structural disturbance of most grain boundaries is limited to a layer of about one nanometer thickness, this limits the applicability of the theory to polycrystals with grain size larger than about one micron.

Information about the state of dislocation is carried in the point statistics only so far as they naturally contain statistical information about the net Burgers vector content through the dislocation density tensor. The specific character of dislocation populations present in the material is not required by the theory, nor is it measured by the experimental technique.

The new method of orientation imaging microscopy (OIM) has been used to obtain reliable one- and two-point statistical data of placement of lattice orientation. OIM relies upon rapid indexing of electron backscattered (Kikuchi) diffraction patterns to obtain lattice orientation from numerous locations over polished flat surfaces sectioned from polycrystalline samples (Adams et al., 1993).

Data obtained on each individual section plane can be used to estimate the oneand two-point statistics, $f_{1}(g)$ and $f_{2}\left(g, g^{\prime} / \mathbf{r}\right)$ defined by the relations

$$
\begin{aligned}
& \mathrm{d} N / N=f_{1}(g) \mathrm{d} g, \\
& \mathrm{~d} N / N=f_{2}\left(g, g^{\prime} / \mathbf{r}\right) \mathrm{d} g \mathrm{~d} g^{\prime} .
\end{aligned}
$$

Here $g$ is the lattice orientation at a random point, and $g^{\prime}$ denotes the lattice orientation at a position $\mathbf{r}$ from the random point $\left(g, g^{\prime} \in \mathrm{O}(3)\right)$. In the case of the one point statistics, $\mathrm{d} N / N$ represents the number fraction of random trials (probe points) which fall in an infinitesimal region of invariant measure $\mathrm{d} g$ about the lattice orientation $g$. Thus $f_{1}(g)$ is the probability density function, better known (although misnamed) as the orientation distribution function ODF (Bunge, 1982).

For two-point experiments $\mathrm{d} N / N$ denotes the number fraction of trials in which a random vector $\mathbf{r}$ (fixed length and direction) finds lattice orientation in the neighbourhood of measure $\mathrm{d} g^{\prime}$ about $g^{\prime}$ at its head, and lattice orientation in the neighbourhood of measure $\mathrm{d} g$ about $g$ at its origin (randomly located). The function $f_{2}\left(g, g^{\prime} / \mathbf{r}\right)$ has been called the two-point orientation correlation function (OCF) (Adams et al., 1987).

Since most polycrystalline materials are substantially opaque to electrons, experiments must access data from multiple section planes in order to cover the homogeneous space of physically distinctive directions for $\mathbf{r}$. In this work, seven section planes were selected which provide nearly uniform coverage of directions in $D_{2 \mathrm{~h}} / S^{2}$ with an angular resolution of approximately $\pi / 14$. $\left(D_{2 h} / S^{2}\right.$ is the appropriate homogeneous space for polycrystals processed by rolling to obtain orthotropic properties.) Three-point statistical data, i.e. $f_{3}\left(g, g^{\prime}, g^{\prime \prime} / \mathbf{r}, \mathbf{r}^{\prime}\right)$, can also be obtained from sectioning 
of the same type if sufficient sections are accessible; three-point statistics were not recovered in the present work. Higher-order statistical information ( $n$-point statistics, $n>3$ ) demands more complete three-dimensional data, which will typically require difficult tomographic sectioning procedures beyond the scope of this investigation.

The relationship between average tensors, including correlation tensors and the loworder statistics on lattice orientation is straightforward for statistically homogeneous polycrystals. Let $T^{\circ}$ denote an arbitrary reference (material) tensor, and $g T^{\circ}$ a new tensor, related to the reference one by the action of the rotation/inversion specified by $g \in \mathrm{O}(3)$. The average tensor, $\langle T\rangle$, is obtained from $T^{\circ}$ and $f_{1}(g)$ by the relation

$$
\langle T\rangle=\int f_{1}(g) g T^{\circ} \mathrm{d} g,
$$

where the integration is over the entire fundamental zone of physically distinctive lattice orientations, $\mathrm{O}(3) / G$, where $G$ is the point symmetry-subgroup associated with the crystal lattice. The two-point correlation tensor, $\langle T(\mathbf{x}) T(\mathbf{x}+\mathbf{r})\rangle$, is obtained from $T^{\circ}$ and $f_{2}\left(g, g^{\prime} / \mathbf{r}\right)$ by the expression

$$
\langle T(\mathbf{x}) T(\mathbf{x}+\mathbf{r})\rangle=\iint f_{2}\left(g, g^{\prime} / \mathbf{r}\right) g T^{\circ} g^{\prime} T^{\circ} \mathrm{d} g \mathrm{~d} g^{\prime} .
$$

As before, the integrations are to be conducted over the entire fundamental zone of each variable.

\subsection{Characterization of the samples used in the experiments}

The material studied was oxygen-free electronic (OFE) alloy 101 copper plate $(99.99 \%$ pure). This material has face-centered-cubic crystal structure with the point symmetry-subgroup $\mathrm{O}_{h}$. As received the material was treated to $1 / 2$ hard temper corresponding to a rolling reduction of $20.7 \%$ (true strain of 0.232 ). This material was subsequently annealed at $600^{\circ} \mathrm{C}$ for $1.5 \mathrm{~h}$ under an argon atmosphere, followed by furnace cooling. Seven section planes were cut from the annealed material using a slow-speed diamond saw.

The basic cubic copper crystal had the following values:

$$
\begin{aligned}
C_{1111} & =168.4 \mathrm{GPa} \\
C_{1212} & =75.39 \mathrm{GPa} \\
C_{1122} & =124.4 \mathrm{GPa} \\
\kappa & =137.07 \mathrm{GPa} \text { (the bulk modulus). }
\end{aligned}
$$

Each section was mounted in a $3.2 \mathrm{~cm}$ diameter thermosetting mount and hand polished using progressively finer grit paper and diamond paste. Once a bright finish was obtained, the samples were electropolished with an electrolyte consisting of methanol $(375 \mathrm{ml})$, cupric nitrate $(125 \mathrm{gm})$ and nitric acid $(12 \mathrm{ml})$.

OIM was used to construct grain maps for each of the seven sample sections using established procedures (Adams et al., 1993). A one-quarter section of the grain map 


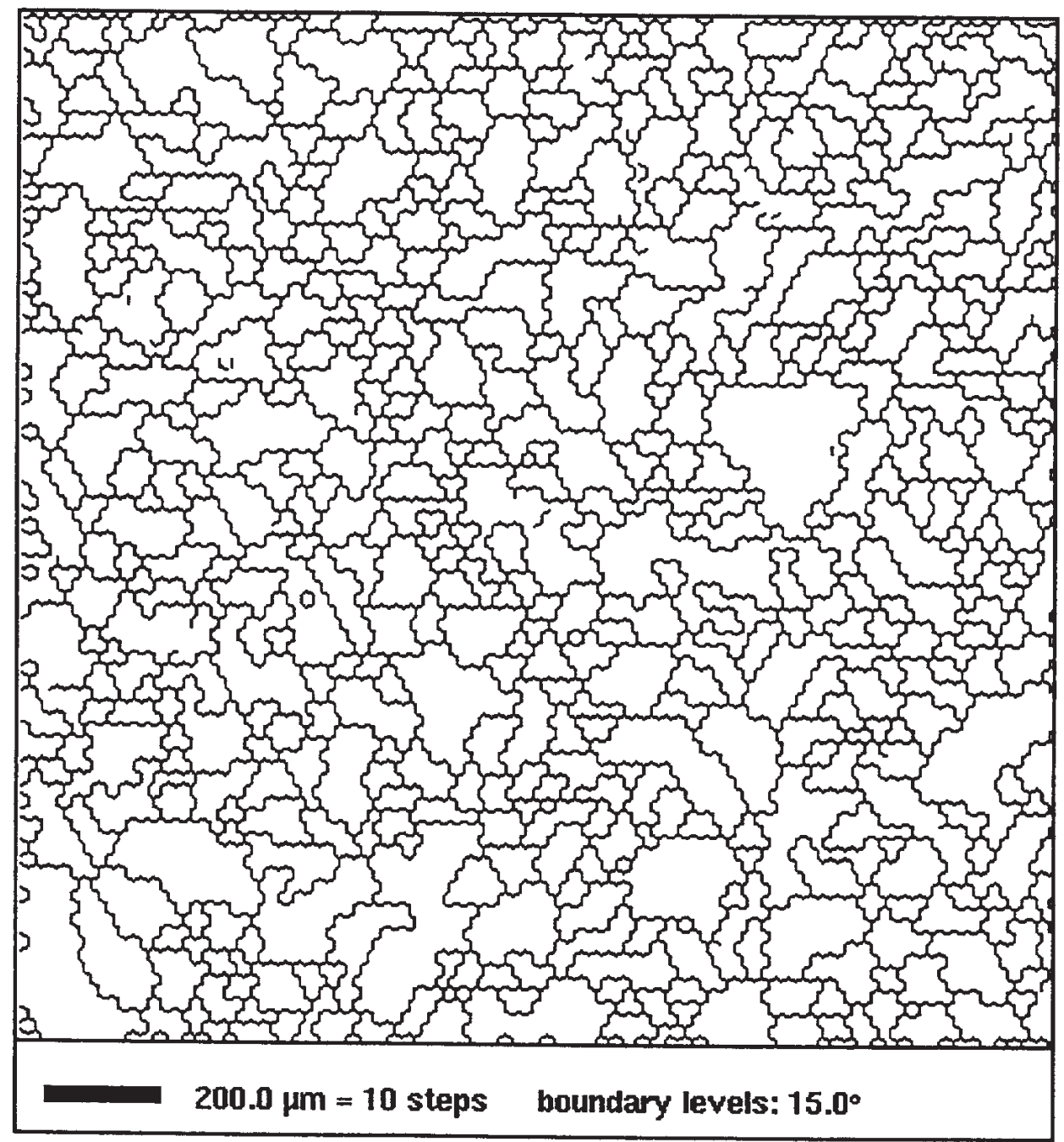

Fig. 1. Reconstructed grain map for section 7. Lines denote orientation differences of $15^{\circ}$ or more.

for a sample is shown in Fig. 1. [Figures 1-5 and Table 1 are taken from Mason (1994).] The grains are delineated by lines separating regions distinguished by at least $\pi / 12$ of lattice disorientation. Approximately 30,000 single orientation measurements have been used to construct a grain map for each of the seven sections. Each section plane exposes approximately 4,000 grains and 12,000 grain boundaries.

The grain size was estimated from the average number of intersections per unit of length of linear test lines applied to the grain maps in the three principal processing directions: rolling direction (RD), transverse direction (TD) and normal direction (ND). Three planes were examined : the rolling-transverse (RT), rolling normal (RN) and the transverse normal (TN). The results are given in Table 1. Clearly the microstructure is "equiaxed" based upon the uniformity of grain size in each direction and from the nominal uniformity of the standard deviation of grain sizes, although a 
Table 1. Average grain size and standard deviation on $R N, T N$ and $R T$ planes

\begin{tabular}{lll}
\hline Section & $\begin{array}{l}\text { Direction and } \\
\text { average grain size }\end{array}$ & $\begin{array}{l}\text { Standard deviation } \\
\text { of grain size }\end{array}$ \\
\hline 1-RN plane & RD $-65.20 \mu \mathrm{m}$ & RD $-41.87 \mu \mathrm{m}$ \\
$4-T N$ plane & ND $-60.75 \mu \mathrm{m}$ & ND $-28.51 \mu \mathrm{m}$ \\
$7-$ RT plane & TD $57.10 \mu \mathrm{m}$ & TD $-31.83 \mu \mathrm{m}$ \\
& ND $-60.75 \mu \mathrm{m}$ & RD $-37.62 \mu \mathrm{m}$ \\
& RD $-59.79 \mu \mathrm{m}$ & TD $-35.06 \mu \mathrm{m}$ \\
\hline
\end{tabular}

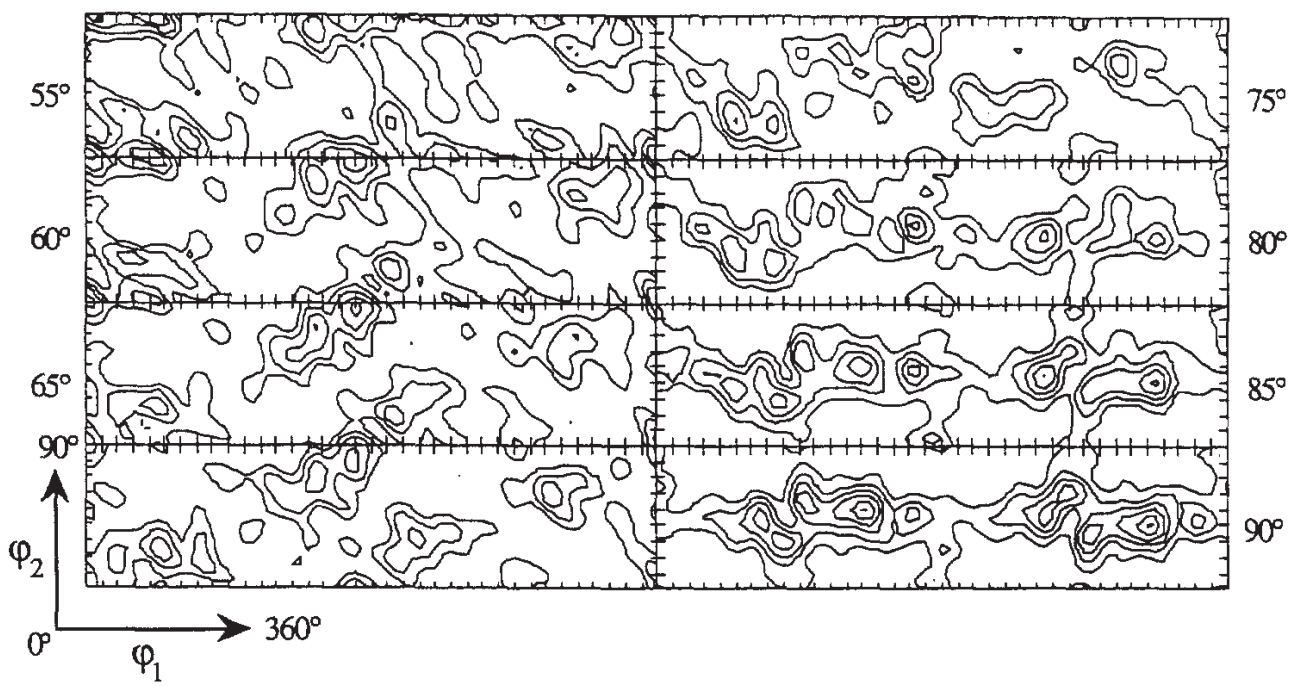

Fig. 2. Orientation distribution function for section 7. Contour levels are drawn at $1,1.5,2,2.5,3$ and 3.35 times random. The maximum peak value was 3.375 .

slight preferential elongation of grains in the RD direction may be indicated from the data.

The ODF, $f_{1}(g)$ is plotted in Fig. 2. The set of single orientation data from the seventh section plane has been used to estimate the function shown in Fig. 2. The ODFs were determined for each of the other section planes; these show negligible differences from the one obtained for the seventh section plane. The parameters used to span $\mathrm{O}(3) / \mathrm{O}_{\mathrm{h}}$ are the Euler angles $\phi_{1}, \phi, \phi_{2}$ defined according to the standard of Bunge (1982). The orthotropic processing symmetry $\left(D_{2 \mathrm{~h}}\right)$ has not been imposed in presenting the data, but is clearly indicated in the natural occurence in the data of the eight expected mirror planes and two-fold rotational axes associated with $D_{2 \mathrm{~h}}$.

The maximum strength of the ODF was found to be 3.375 times random, which is rather weak texture owing to the severity of the annealing procedure. The thermal treatment was chosen with the object in mind of removing as much of the stored strain energy of the material as possible by means of recystallization and some grain growth. Stored dislocation strain energy can affect elastic constant measurements of 
the type used to obtain measurements on the present samples (Ledbetter, private communication). This effect is reduced to negligible levels by the treatment used. The recrystallization process leads to a weakening of the texture in the material, however, tending towards a more isotropic ODF. This weakening of the ODF proved to have the undesirable consequence that the material demonstrated only rather small deviations from isotropy. The main peaks of the distribution are consistent with those observed for rolled copper sheet (Bunge, 1982).

It is hardly practical to explore the details of the OCF in any systematic fashion since it lies on a nine-dimensional manifold (three parameters for each lattice orientations and three parameters describing the separation vector $\mathbf{r}$ ). Here we present a few three-dimensional sections of the measured OCF taken in a particular way. The misorientation distribution (MDF), $m(\Delta g / \mathbf{r})$ is defined from the OCF in the following way

$$
m(\Delta g / \mathbf{r})=\int f_{2}(g, \Delta g \cdot g / \mathbf{r}) \mathrm{d} g
$$

where the integration is taken over the homogeneous space $\mathrm{O}(3) / \mathrm{O}_{\mathrm{h}}$ of physically distinctive orientations of the cubic lattice. For fixed $\mathbf{r}$ the MDF lies on the doubly symmetric space $\mathrm{O}_{\mathrm{h}} \backslash \mathrm{O}(3) / \mathrm{O}_{\mathrm{h}}$ of distinctive misorientations of the cubic lattice. The MDF describes the distribution of disorientations of the underlying lattices of two random points separated by the specified vector $\mathbf{r}$ in the microstructure. Since many intrinsic (thermodynamic and kinetic) properties of grain boundaries are known to be sensitive to lattice disorientation (Sutton and Balluffi, 1995), the MDF is a particularly important projection of the OCF.

Using the Euler-angle parameters defined by Zhao and Adams (1988), the MDF for annealed OFE copper samples has been plotted for $\mathbf{r} /$ RD in Figs 3-5. In Fig. 3 the magnitude of $\mathbf{r},|\mathbf{r}|$, is selected to be $|\mathbf{r}|=d$ where $d$ is the average grain diameter ( $\sim 60$ microns). Thus Fig. 3 describes mainly the nearest neighbor disorientations (i.e. separated by only one grain boundary). In Figs 4 and $5,|\mathbf{r}|=3 d$ and $7 d$, respectively. Similar MDFs were explored for directions other than RD in the samples. Their distributions are found to be similar, although in general the peak values of the MDF are somewhat weaker than the values found in the RD direction. In each of these figures the strongest feature is the peak near the center of the $\phi=72^{\circ}$ section, which is associated with $\Sigma 3$ disorientations in the coincidence site lattice (Adams et al., 1990). Also prominent are the $\Sigma 9$ disorientations. The strength of the $\Sigma 3$ peak is seen to decrease from 12 to 1 times random as $\mathbf{r}$ increases its length from $1 d$ to $7 d$ along RD.

In fact, it is observed that at $|\mathbf{r}|$ approximately equal to $7 d$, the two-point statistics decouple such that

$$
f_{2}\left(g, g^{\prime}|\mathbf{r}:| \mathbf{r} \mid>7 d\right)=f_{1}(g) f_{1}\left(g^{\prime}\right) .
$$

This decoupling is particularly evident on one-dimensional projections of the OCF which describe the distributions of disorientation angles between pairs of lattices separated by fixed $|\mathbf{r}|$. Figure 6 illustrates these distributions for $|\mathbf{r}|=1 d, 3 d$ and $7 d$. It is noted that at $|\mathbf{r}|=7 d$ the distribution very closely approximates the known 

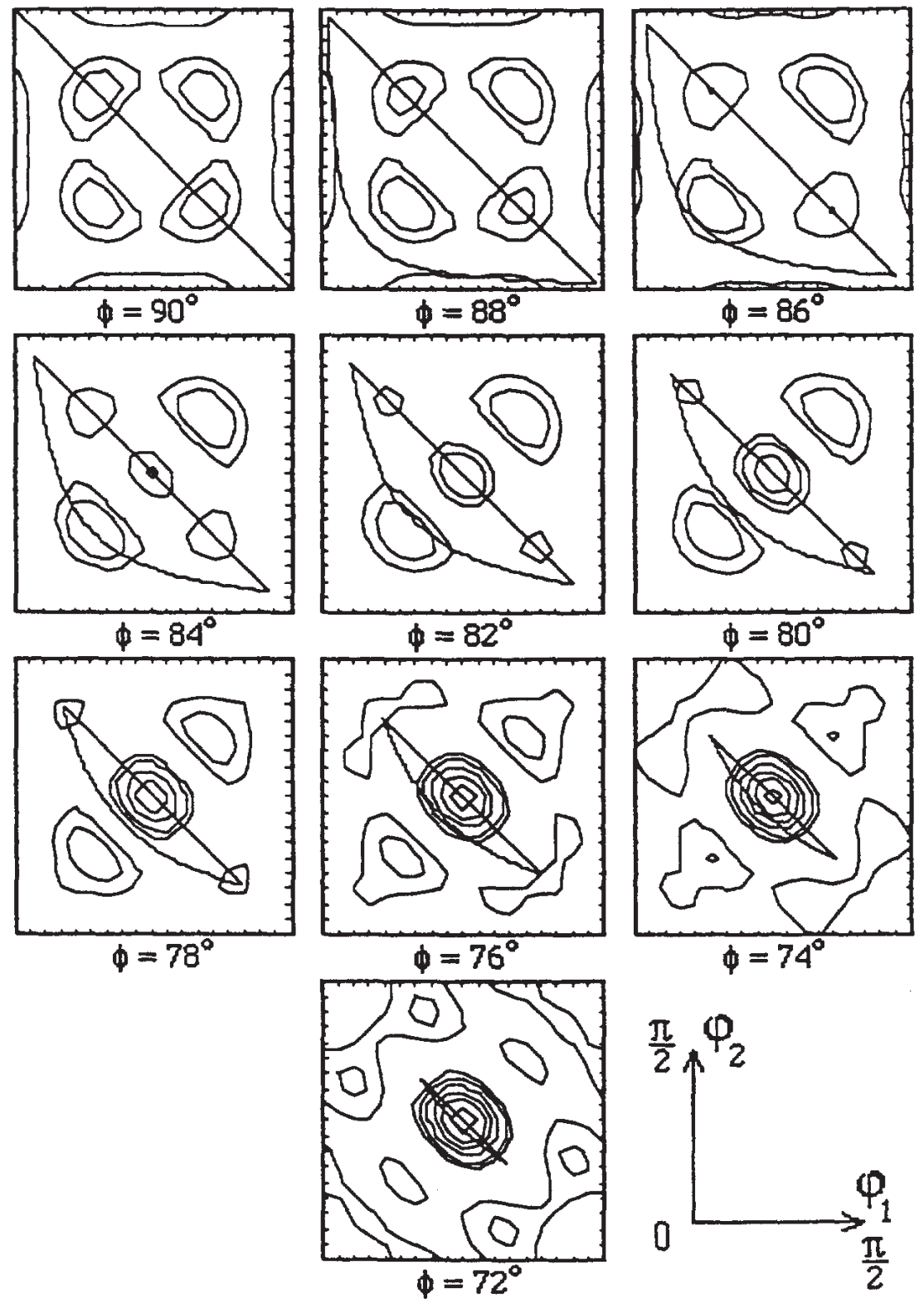

Fig. 3. The MDF for pairs of single orientations that are one average grain diameter apart. Misorientations of $15^{\circ}$ or less have been excluded. Contour levels are $1,2,4,6,8,10$ and 12 times random.

theoretical distribution for random placement of cubic crystals (Mackenzie, 1958, 1964).

We elected to not extend the experimental measurements to determine the threepoint statistical information. Although seven section planes prove to cover $D_{2 h} \backslash S^{2}$ with an angular resolution of about $\pi / 14$, a substantially larger number of sections is required to properly cover the double space $D_{2 h} \backslash S^{2} D_{2 h} \backslash S^{2}$ in order to access three- 


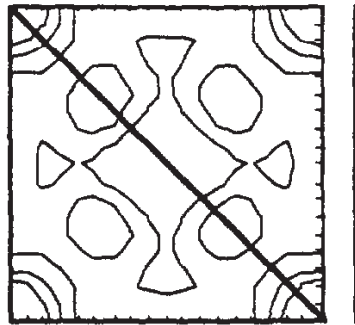

$\phi=90^{\circ}$

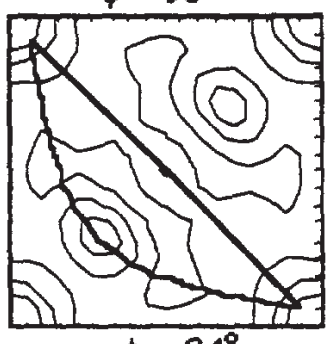

$\phi=84^{\circ}$
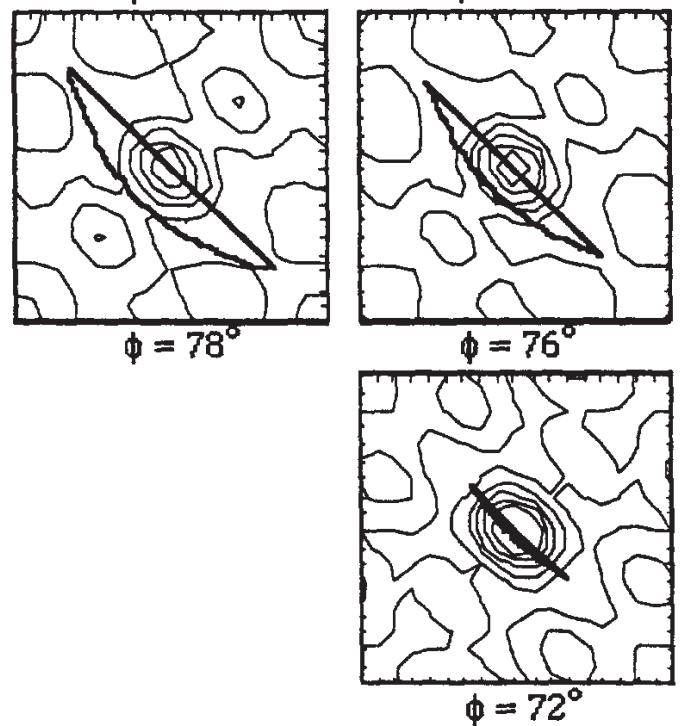
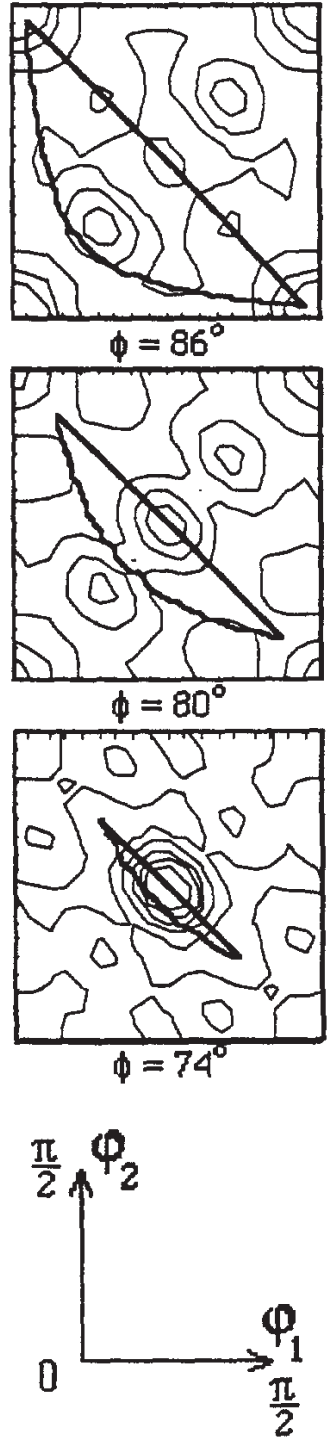

Fig. 4. The MDF for pairs of orientations three average grain diameters apart in the rolling direction of the sample. Contour levels are $0.3,0.6,0.9,1.2,1.5,1.8$ and 2.1 . The maximum value of 2.75 occurs at the center of the $\phi=72$ section. Note that all misorientations below $15^{\circ}$ have been overlooked in this calculation to show other significant features of the distribution.

point data with the same angular resolution. For this reason, consideration of the three-point statistics has been left for a future effort.

\subsection{Experimental determination of the effective constants $\mathrm{C}_{\mathrm{ij \textrm {km }}}^{*}$}

The macroscopic elastic response of the copper specimens was performed at the National Institute of Standards and Technology by H. M. Ledbetter. The measurements were performed on a prismatic specimen milled from the center of the annealed 


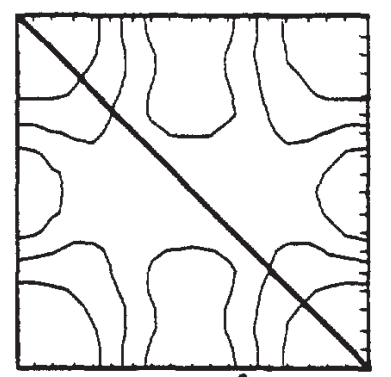

$\phi=90^{\circ}$

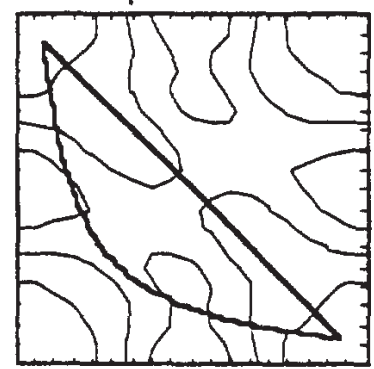

$\phi=84^{\circ}$

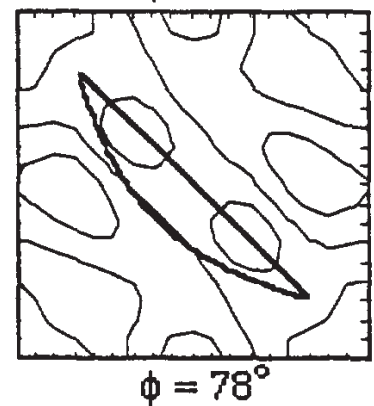

$=78^{\circ}$

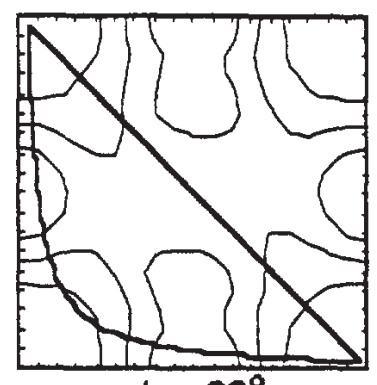

$\phi=88^{\circ}$

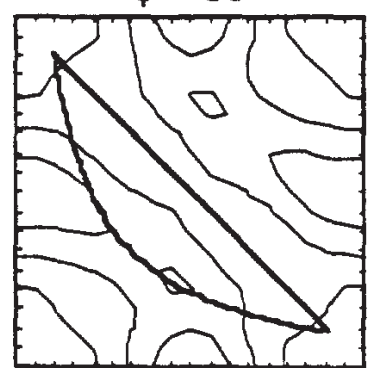

$\phi=82^{\circ}$

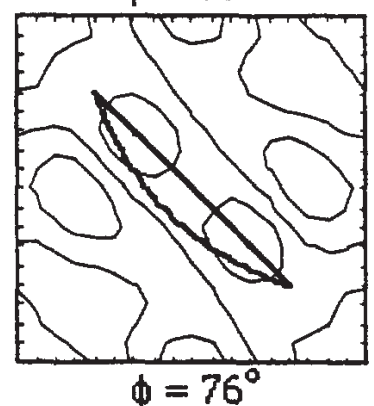

$\phi=76^{\circ}$

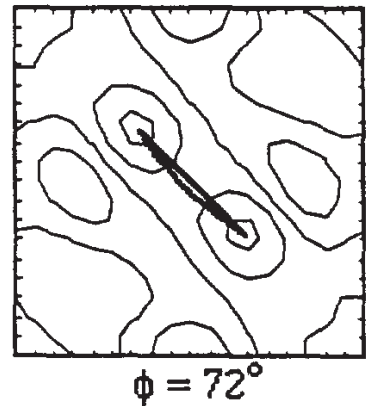

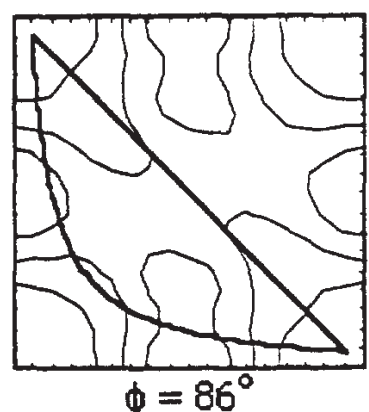

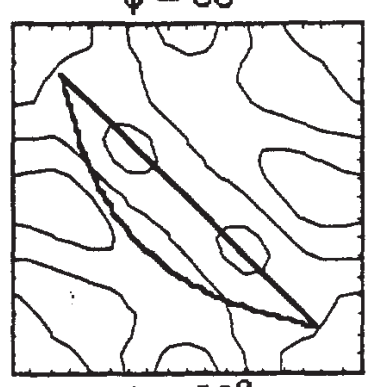

$\phi=80^{\circ}$
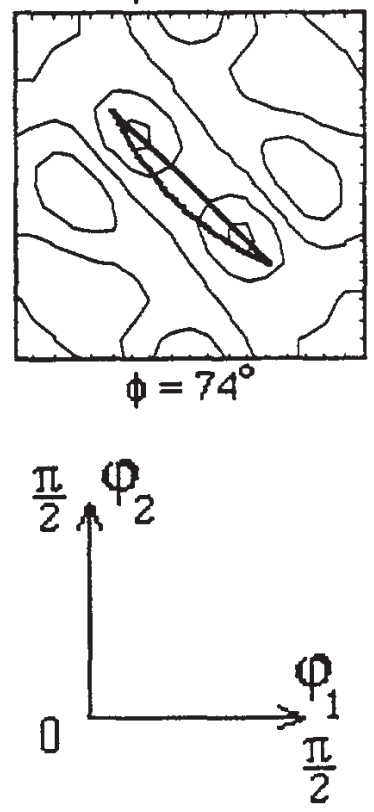

Fig. 5. The MDF for pairs of orientations seven average grain diameters apart in the rolling direction of the sample. Contour levels are $0.95,1.0,1.05$ and 1.1 . Maximum value is 1.115 and the minimum value is 0.903 .

strip discussed in Section 5.1. The results were calculated from pulse-echo acoustic measurements. The measurements are discussed at length by Mason (1994). The values for the measured effective elastic constants are given in Table 2 under the heading "Exp.". 
(a)

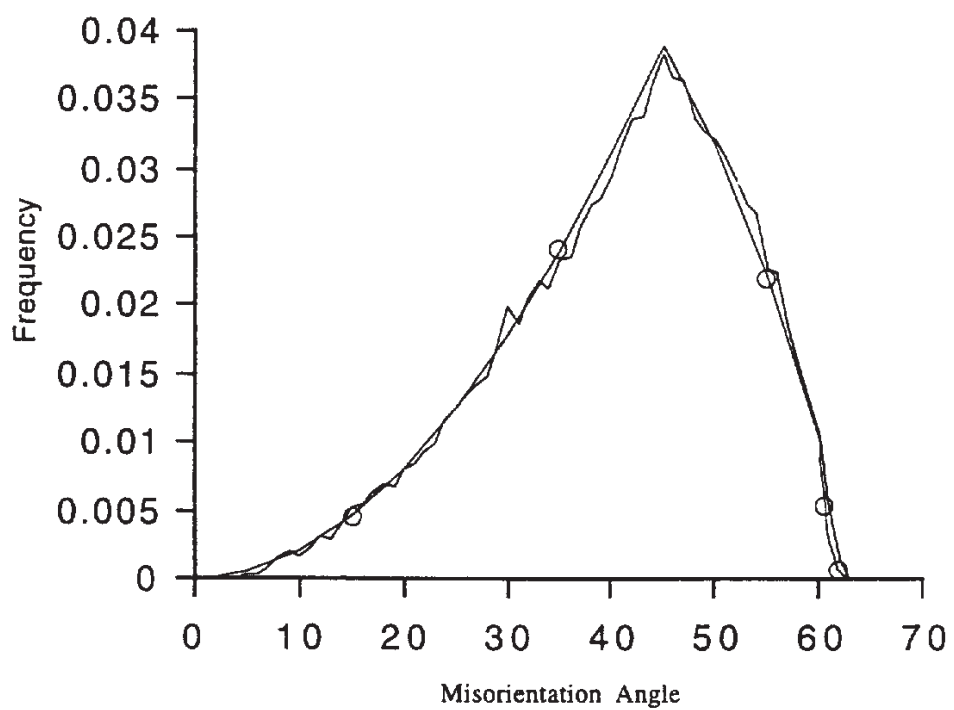

(b)

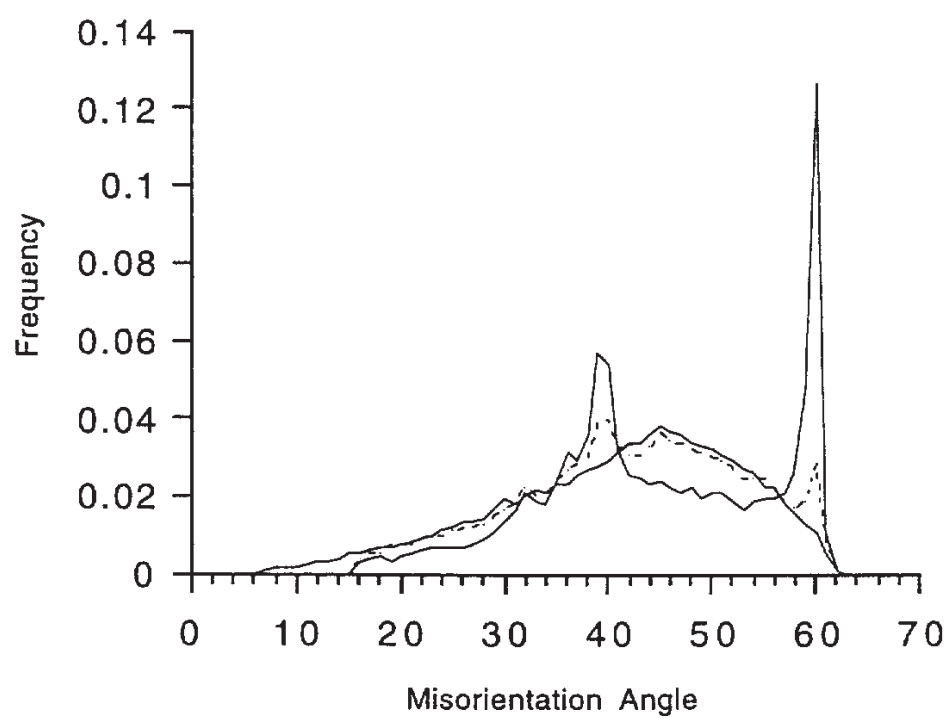

Fig. 6. Distribution of disorientation angles for the OFE copper sample. (a) Comparison of the experimental disorientation distribution for $|\mathbf{r}|=7 d$ with the theoretical random disorientation distribution (piecewise smooth). (b) Comparison of the experimental disorientation distributions for $|\mathbf{r}|=1 d$ (large peaks at $40^{\circ}$ and $\left.60^{\circ}\right),|\mathbf{r}|=3 d$ (dashed curve with smaller peaks at $40^{\circ}$ and $60^{\circ}$ ), and $|\mathbf{r}|=7 d$ [compare with (a)].

\subsection{Perturbation results}

The perturbation solution is given by the expression in (16). The integrals were evaluated and the results are given in Table 2. Included in Table 2 were also the Voigt and Reuss bounds and the bounds considered in Section 4.1.3. The bounds will be considered in more detail in the next section. In this section we are concerned with the columns headed "Pert." (the perturbation solution) and "Exp." (the experimental results discussed in Section 5.3). As we see, the agreement is good. It is considerably 
Table 2.

\begin{tabular}{lcccccc}
\hline Comp. & Voigt & UB & Pert. & Exp. & LB & Reuss \\
\hline$C_{1111}^{*}$ & 209.8 & 202.4 & 202.0 & 199.0 & 197.8 & 192.2 \\
$C_{2222}^{*}$ & 211.0 & 204.1 & 203.7 & 200.7 & 199.5 & 193.3 \\
$C_{3333}^{*}$ & 210.7 & 203.9 & 203.4 & 200.9 & 199.2 & 193.0 \\
$C_{1212}^{*}$ & 54.54 & 49.35 & 48.97 & 46.42 & 45.83 & 39.97 \\
$C_{1313}^{*}$ & 54.83 & 49.62 & 49.30 & 47.36 & 46.14 & 40.24 \\
$C_{2323}^{*}$ & 53.65 & 48.35 & 48.02 & 47.66 & 44.85 & 39.19 \\
\hline
\end{tabular}

better than the Voigt bound (which corresponds to the average value of the modulii) or the Reuss bound. However, this lowest-order perturbation result is not close enough to the experimental results to be completely satisfactory. We expect that, if the next term in the perturbation series was calculated, better agreement with experiment would be obtained. As we stated in the previous section the experimental data are not available for this calculation.

As we stated in Section 4.1.4, we expected the $M_{i j}$ contribution to be dominant in (16) since the material is close to isotropic. This was indeed the case, with the $M_{i j}$ contribution being more than $94 \%$ of the total contribution. For example, for $C_{1111}^{*}$, the $M_{i j}$ contribution is $95 \%$ of the total.

\subsection{Upper and lower bound results}

In Table 2, we list upper and lower bound results for the Voigt and Reuss bounds [Section 4.1.1, equations (24) and (25)] and for bounds using two-point correlation information [Section 4.1.3, equations (39) and (40)]. At the present time we have not calculated the bounds using the three-point correlation data [Section 4.1.2, equations (30), (34) and (37)].

The Voigt and Reuss bounds are the bounds obtained by using the constant trial functions described in Section 4.1.1. As we see from Table 1, they are rather weak bounds and deviate by about $5-15 \%$ from the experimental results.

Better bounds are obtained by introducing two-point correlation data into the bounds. This may be done by modifying the bounds given in Section 4.1.2 as was done in Section 4.1.3 or by using the variational principles used by Hashin and Shtrikman (1962). For the shear modulii these bounds are given by (39) and (40). The expressions for the constants $C_{1111}^{*}, C_{2222}^{*}$ and $C_{3333}^{*}$ are given in Mason (1994). These results are presented in Table 2 under the headings UB and LB. Details of the calculation are given in Mason (1994). This bound spread is much smaller than the Voigt and Reuss bound spread. As in the perturbation case, Mason (1994) shows that the contribution of the shape of the two-point correlation function is small compared to contribution from the singularity.

Finally, we note again that, when the fluctuations are very small the UB and LB bounds coincide with the perturbation solution. In the example presented, the fluctuations are large enough so that the bounds diverge from the perturbation 
solution. However, the perturbation solution is very close to the upper bound. Interestingly, the experimental results lie close to the lower bound.

The material we have chosen is not too far from isotropic and the effect of the shape of the two-point correlation function is small. However, the purpose of this paper was to demonstrate that both the anisotropy and the effect of the shape of the two-point correlation function, although not large, could be measured and compared to theory. The reason for the small deviation from isotropy was discussed in Section 5.2. In future work we intend to measure samples with larger deviations from isotropy.

\section{SUMMARY}

We have studied the problem of using the statistical properties of the microstructure in order to determine information about the elastic constants of an orthotropic polycrystal. We make use of two-point correlation information although in preparation for future experimental measurements we have derived theoretical expressions using three-point correlation information. Expressions have been derived for the perturbation solution (16), the Voigt and Reuss bounds (25) and bounds containing two- and three-point correlation information (Sections 4.1.2 and 4.1.3).

Experimental results were obtained for OFE alloy 101 copper plate, using orientation-imaging microscopy. The elastic constants were obtained directly by acoustic measurements (Section 5.2). The perturbation and bounding results are summarized in Table 1 for comparison with the acoustic measurements. The Voigt and Reuss bounds are not close enough to provide a good estimate of the measured acoustic values. Including two-point information into the bounds narrows the bound spread considerably.

\section{REFERENCES}

Adams, B. L., Morris, P. R., Wang, T. T., Willden, K. S. and Wright, S. I. (1987) Description of orientation coherence in polycrystalline materials. Acta Metall. 35, 2935-2946.

Adams, B. L., Wright, S. I. and Kunze, K. (1993) Orientation imaging: The emergence of a new microscopy. Met. Trans. A24, 819-831.

Adams, B. L., Zhao, J. and Grimmer, H. (1990) Discussion of the representation of intercrystalline misorientation in cubic materials. Acta Crystall. A46, 620-622.

Beran, M. J. (1968) Statistical Continuum Theories. John Wiley and Sons, Interscience, New York.

Beran, M. J. and McCoy, J. J. (1970) Mean field variations in a statistical sample of heterogeneous linearly elastic solids. Int. J. Solids Structures 6, 1035-1053.

Brown, W. F. (1962) Magnetostatic Principles in Ferromagnetism. North-Holland Publications, Amsterdam.

Bunge, H. J. (1982) Texture Analysis in Materials Science: Mathematical Methods. Butterworths, London.

Hashin, Z, and Shtrikman, S. (1962) A variational approach to the theory of the elastic behavior of polycrystals. J. Mech. Phys. Solids 10, 343-352.

Korringa, J. (1973) Theory of elastic constants of heterogeneous media. J. Math. Phys. 14, 509-513.

Kroner, E. (1967) Elastic modulii of disordered materials. J. Mech. Phys. Solids 15, 319-329. 
Kroner, E. (1986) Statistical modeling. In Modeling Small Deformations of Polycrystals (ed. J. Gittus and J. Zarka), pp. 229-292. Elsevier Applied Science Publishers.

Ledbetter, H. M. and Read, D. T. (1977) Orthorhombic elastic constants of an NbTi/Cu composite superconductor. J. Appl. Phys. 48, 1874-1879.

Mackenzie, J. K. (1958) Second paper on statistics associated with the random disorientation of cubes. Biometrika 45, 229-240.

Mackenzie, J. K. (1964) The distribution of rotation axes in a random aggregate of cubic crystals. Acta Metall. 12, 223-225.

Mason, T. A. (1994) Use of two-point correlation functions in bounding the elastic properties of polycrystals. Ph.D. Thesis, Yale University, New Haven.

McCoy, J. J. (1981) Macroscopic response of continua with random microstructures. In Mechanics Today, Vol. 6 (ed. S. Nemat-Nassar). Pergamon Press, New York.

Sutton, A. P. and Balluff, R. W. (1995) Interfaces in Crystalline Materials, Parts II and III. Clarendon Press, Oxford.

Walpole, L. J. (1987) Orthotropically textured elastic aggregates of cubic crystals. J. Mech. Phys. Solids 35, 497-517.

Willis, J. R. (1981) Variational and related methods for the overall properties of composites. Advances in Applied Mechanics, Vol. 21 (ed. Chia-Shun Yih). Academic Press, New York.

Zhao, J. and Adams, B. L. (1988) Definition of an asymmetric domain for intercrystalline misorientation in cubic materials in the space of Euler angles, Acta Crystall. A44, 326-336. 